\title{
OPEN Measurement of bunch length and temporal distribution using accelerating radio frequency cavity in low-emittance injector
}

\author{
Ji-Gwang Hwang ${ }^{1}$, Tsukasa Miyajima ${ }^{2 \bowtie}$, Yosuke Honda² \& Eun-San Kim ${ }^{3 凶}$
}

We demonstrate an experimental methodology for measuring the temporal distribution of picosecond level electron bunch with low energy using radial electric and azimuthal magnetic fields of an accelerating ( $T M_{01}$ mode) radio frequency (RF) cavity that is used for accelerating electron beams in a linear accelerator. In this new technique, an accelerating RF cavity provides a phase-dependent transverse kick to the electrons, resulting in the linear coupling of the trajectory angle with the longitudinal position inside the bunch. This method does not require additional devices on the beamline since it uses an existing accelerating cavity for the projection of the temporal distribution to the transverse direction. We present the theoretical basis of the proposed method and validate it experimentally in the compact-energy recovery linac accelerator at KEK. Measurements were demonstrated using a 2-cell superconducting booster cavity with a peak on-axis accelerating field $\left(E_{0}\right)$ of $7.21 \mathrm{MV} / \mathrm{m}$.

Future accelerators, such as X-ray free-electron lasers $(\mathrm{FEL})^{1-7}$, energy recovery linacs $(\mathrm{ERL})^{8-11}$, and linear colliders $^{12-15}$, are aiming at high-brightness and high-brilliance. This can be accomplished by a high-performance injector that can produce electron beams with an ultra-low emittance in 6-D phase space. This scientific motivation stimulates the development of high-power and low-emittance photo-injectors ${ }^{16-23}$ that consist of a superconducting linear accelerator capable of producing a continuous stream of electron bunches at repetition rates of a few MHz. These injectors also open new horizons for many applications in the physical sciences, materials science, chemistry, health, information technology and security ${ }^{24-28}$. For these injectors, measurements of the temporal profiles of electron beams are important for achieving the desired performance. Many instruments and technologies, such as streak cameras ${ }^{29-31}$, transverse deflecting cavities ${ }^{32-36}$, electro-optic probes ${ }^{37,38}$, Terahertz streaking $^{39,40}$ and devices based on the coherent property of synchrotron radiation ${ }^{41-48}$, have been devised for measuring and monitoring the longitudinal parameters of picosecond (ps) and sub-picosecond electron bunches, have been applied in accelerators. These methods, however, require construction space for installing complex and expensive equipment, such as sophisticated streaking devices with highly accurate timing systems, high-quality optical elements, or deflecting cavities with high power sources.

We propose a novel technique for measuring few-ps level bunch lengths by using radial electric and azimuthal magnetic fields of an accelerating $\left(\mathrm{TM}_{01}\right.$ mode) $\mathrm{RF}$ cavity which is well known for the source of second-order ponderomotive focusing force ${ }^{49}$. The advantage of this method is that it only requires a corrector magnet installed upstream in an accelerating RF cavity for manipulating a beam offset inside the cavity, and a profile monitor installed downstream in the cavity for measuring a transverse beam distribution. Therefore, injectors of accelerators worldwide, which have low energy beams with an accelerating RF cavity, can use this method for measuring the longitudinal distribution as well as few-ps bunch lengths without installing any specialized devices. We present the theoretical basis and estimation of the resolution for our method. In addition, the projection of the longitudinal distribution (which is a semi flat-top distribution with $13.51 \mathrm{ps}(\mathrm{rms})$ ) to the transverse direction has been demonstrated at the $\mathrm{CERL}^{50}$ which has 2-cell superconducting radio frequency (SRF) cavities in an injector. The result of the measurement for a bunch length of $3.3 \mathrm{ps}$ ( $\mathrm{rms}$ ), which is closest to the resolution of the system, is also verified experimentally.

\footnotetext{
${ }^{1}$ Helmholtz-Zentrum Berlin (HZB), Albert-Einstein straße 15, 12489 Berlin, Germany. ${ }^{2}$ KEK, High Energy Accelerator Research Organization, 1-1 Oho, Tsukuba, Ibaraki 305-0801, Japan. ${ }^{3}$ Department of Accelerator Science, Korea University Sejong Campus, Sejong 339-700, South Korea. ${ }^{\varpi}$ email: tsukasa@post.kek.jp; eskim1@ korea.ac.kr
} 

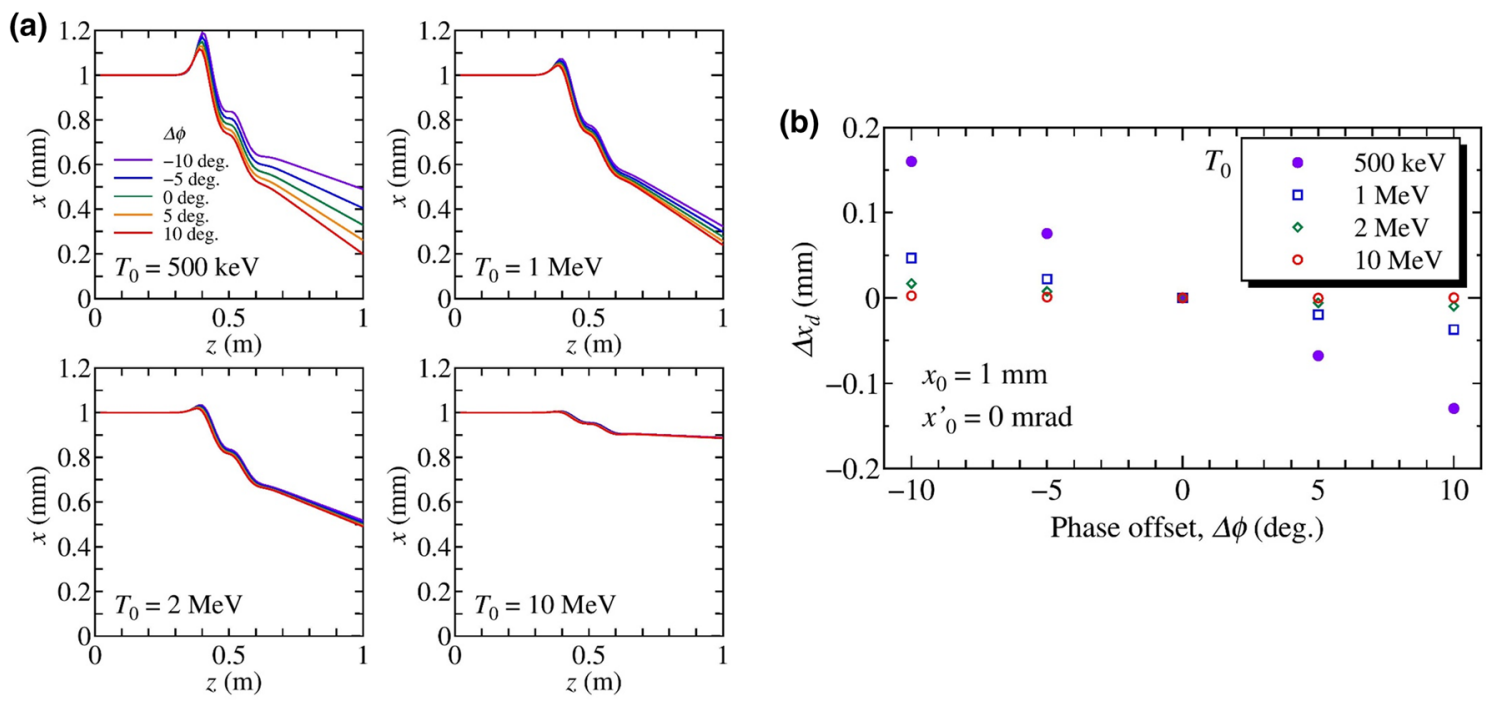

Figure 1. (a) Beam trajectories as a function of $\Delta \phi=\omega \Delta t$ with the initial condition of $x_{0}=1 \mathrm{~mm}$ and $x_{0}^{\prime}=0 \mathrm{mrad}$. The initial kinetic energies are $T_{0}=500 \mathrm{keV}, 1 \mathrm{MeV}, 2 \mathrm{MeV}$ and $10 \mathrm{MeV}$, respectively. (b) Relative deflected positions at the end of the beamline as a function of $\Delta \phi$. The relative position is calculated from the position for the on-crest phase, $\Delta \phi=0$.

\section{Methods}

Our proposed method uses the time-dependent radial electric and azimuthal magnetic fields component of the $\mathrm{TM}_{01}$ mode of an accelerating cavity to project the temporal distribution to a transverse direction. The accelerating $\mathrm{TM}_{01}$ mode only has longitudinal fields at the on-axis so they cannot be used to deflect the beam when electron bunches passes the center of an accelerating cavity. However, the radial electric and azimuthal magnetic fields, which can provide a phase-dependent transverse kick to electron beams, arise at the off-axis of the cavity ${ }^{51}$. Using the paraxial approximation, the equation of motion with the maximum acceleration phase (on-crest phase) is given by

$$
\frac{d p_{r}}{d t}=e\left(E_{r}-\beta c B_{\theta}\right)=-\frac{e r}{2}\left[\frac{\partial E_{m}(z)}{\partial z} \sin \left(\omega(t+\Delta t)-\phi_{m}\left(T_{0}\right)\right)+\frac{\beta \omega}{c} E_{m}(z) \cos \left(\omega(t+\Delta t)+\phi_{m}\left(T_{0}\right)\right)\right],
$$

where $\beta=v / c$, and $E_{r}$ and $B_{\theta}$ are the radial electric field and azimuthal magnetic fields, respectively, $E_{z}=E_{m}(z) \sin \left(\omega t+\phi_{0}\right), \omega=2 \pi f$ where $f$ is the cavity frequency, $\phi_{0}$ is a phase offset that adjusts the acceleration phase, $\phi_{m}\left(T_{0}\right)$ is the on-crest phase at the initial kinetic energy $T_{0}, r$ is the beam offset, and $\Delta t$ is the time difference from $t=0$ at the entrance of the beamline. Equation (1) indicates that the transverse force is a linear function of $r$ around the $z$-axis. Therefore, we can describe the transverse motion using the transfer-matrix formalism, which is given by $x_{1}=M x_{0}$. Here $x_{0} \equiv\left(x_{0}, x_{0}^{\prime}\right)^{T}$ and $x_{1} \equiv\left(x_{1}, x_{1}^{\prime}\right)^{T}$ are the initial and final coordinates in the horizontal phase-space at the entrance and exit of the beamline, respectively, $x^{\prime} \equiv d x / d z$, and $M$ is a $2 \times 2$ transfer matrix. The equation of motion for the vertical axis is equivalent since the cavity is cylindrically symmetric. For high energy beams $(\beta \simeq 1)$, the transfer matrix can be calculated analytically ${ }^{49}$. In contrast, the transverse motion of low energy beams is complicated by the significant changes in the velocity of an electron inside the cavity owing to its low initial kinetic energy and then the trajectories have been numerically simulated using General Particle Tracer (GPT) code ${ }^{52}$ with the electromagnetic field of the 2-cell SRF cavities calculated by using the Poisson/Superfish code ${ }^{53}$ (Figures S1 and S2 in the Supplementary Appendix). The electron with $\Delta t=0$ is accelerated with the on-crest phase. and then the time difference corresponds to the RF phase difference from the on-crest phase, $\Delta \phi=\omega \Delta t$. In this work, degrees are used as the unit of the RF phase and the angular frequency can be represented as $\omega=360 \times f$ in the units of deg./s. A particle tracking simulation was performed for two initial conditions with $x_{0}=1 \mathrm{~mm}$ and $x_{0}^{\prime}=0 \mathrm{mrad}$ at different energies $T_{0}=500 \mathrm{keV}, 1 \mathrm{MeV}, 2 \mathrm{MeV}$ and $10 \mathrm{MeV}$ to evaluate the effect of the time difference on the transverse trajectory. The transverse trajectories and relative deflected positions as functions of the energy and the phase are shown in Fig. 1a,b, respectively.

In the case of a low-energy beam, whose $T_{0}=500 \mathrm{keV}$, the final position $x_{1}$ depends on $\Delta \phi$. This is the origin of the deflection force which is utilized in the proposed method. The transverse forces experienced by the electron when $T_{0}=500 \mathrm{keV}$ and $10 \mathrm{MeV}$ are shown in Fig. S3 (see the Supplementary Appendix). The $\Delta x_{d}(\Delta \phi)$ is a linear function of $\Delta \phi$ around the on-crest phase. In addition, for higher $T_{0}$, the position variation decreases significantly, indicating that the deflection effect is effective for low energy beams. Using on these results, the relative position displacement can be expressed as

$$
\Delta x_{d}(\Delta \phi)=x_{1}(\Delta \phi)-x_{1}(0)=a_{11} \Delta \phi,
$$



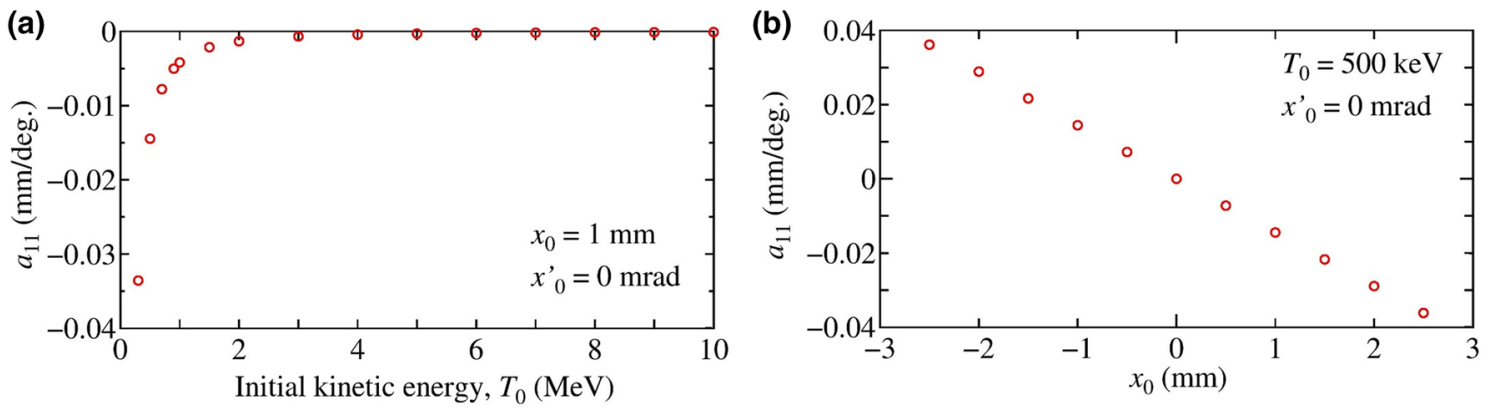

Figure 2. Deflection coefficient $a_{11}$ as functions of (a) the initial kinetic energy $T_{0}$ with initial conditions of $x_{0}=1 \mathrm{~mm}$ and $x_{0}^{\prime}=0 \mathrm{mrad}$, and (b) the initial offset $x_{0}$ with an initial kinetic energy of $500 \mathrm{keV}$ and an initial angle of $x_{0}^{\prime}=0 \mathrm{mrad}$.
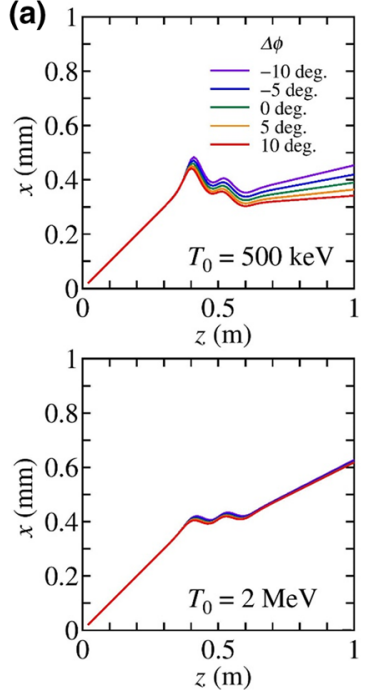

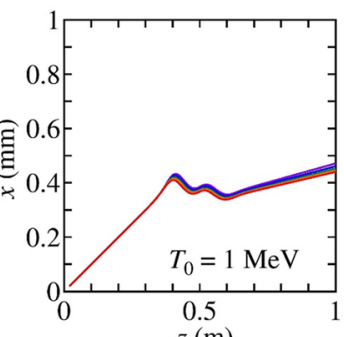

$z(\mathrm{~m})$

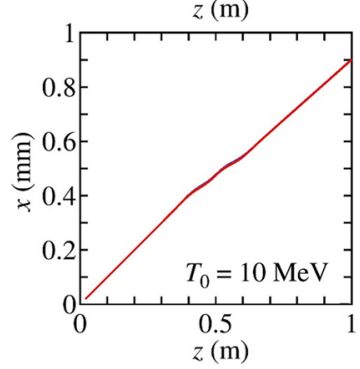

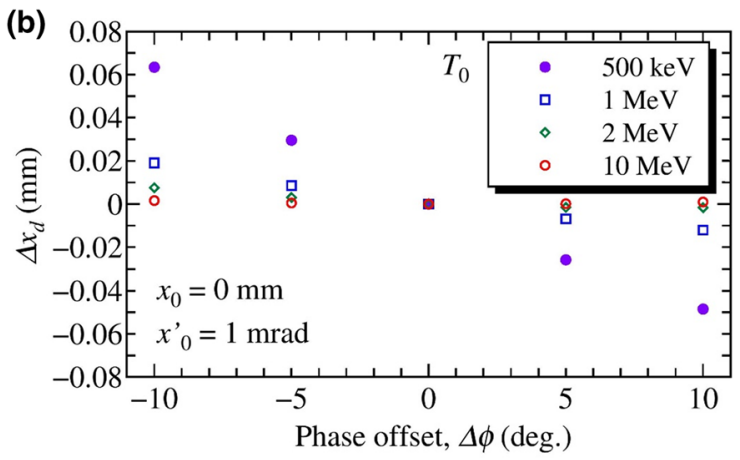

Figure 3. (a) Beam trajectories as a function of $\Delta \phi$ with the initial condition of $x_{0}=0 \mathrm{~mm}$ and $x_{0}^{\prime}=1 \mathrm{mrad}$. The initial kinetic energies are $T_{0}=500 \mathrm{keV}, 1 \mathrm{MeV}, 2 \mathrm{MeV}$ and $10 \mathrm{MeV}$, respectively. (b) Relative deflected positions at the end of the beamline as a function of $\Delta \phi$. The relative position is calculated from the position for the on-crest phase, $\Delta \phi=0$.

where $a_{11}$ is a linear coefficient of $\Delta x_{d}(\Delta \phi)$ which describes the strength of the deflection effect. In order to analyze effects of the initial kinetic energy $T_{0}$ and the initial offset $x_{0}$, the deflection coefficient was simulated as a function of the $T_{0}$ with an initial offset $x_{0}$ of $1 \mathrm{~mm}$ and $x_{0}$, with an initial kinetic energy of $500 \mathrm{keV}$, respectively. The dependence on the initial kinetic energy and offset is shown in Fig. 2.

The simulation results indicate that the deflection coefficient $a_{11}$ depends on the initial offset since the amplitude of transverse electric-field inside the cavity is a linear function of the offset, according to Eq. (1) and the deflection effect is negligible for high energy beams. Therefore, in our proposed method, the deflection effect is effective when $T_{0}<2 \mathrm{MeV}$. Hence, in terms of $x_{0}$ and $\Delta \phi$, the deflection effect becomes:

$$
\Delta x_{d}(\Delta \phi)=d_{11} x_{0} \Delta \phi
$$

where the $d_{11}$ is a coefficient associated with the initial offset. We analyze the effect of the initial angle, $x_{0}^{\prime}$, since it introduces the beam offset while traveling through the cavity. Numerical simulations are performed using the initial condition $x_{0}=0 \mathrm{~mm}$ and $x_{0}^{\prime}=1 \mathrm{mrad}$ to deconvolute the effect of the initial offset. The transverse trajectories, corresponding to $T_{0}=500 \mathrm{keV}, 1 \mathrm{MeV}, 2 \mathrm{MeV}$ and $10 \mathrm{MeV}$, as a function of $\Delta \phi$ were simulated. The results of beam trajectories and relative deflected position at the end of the beamline as a function of $\Delta \phi$ are shown in Fig. 3a,b, respectively. For a low energy beam, the trajectory changes depending on the time difference from the on-crest phase, indicating that the initial angle also affects the deflection force. The $\Delta x_{d}(\Delta \phi)$ is a linear function of $\Delta \phi$ around $\Delta \phi=0$. The relative positional displacement can be expressed as a function of $\Delta \phi$ as

$$
\Delta x_{d}(\Delta \phi)=a_{12} \Delta \phi,
$$

where $a_{12}$ is a coefficient associated with the initial angle. In order to analyze effects of the initial kinetic energy $T_{0}$ and the initial angle $x_{0}^{\prime}$, the deflection coefficient was simulated as functions of the $T_{0}$ with an initial offset $x_{0}^{\prime}$ of $1 \mathrm{mrad}$, and the $x_{0}^{\prime}$ with an initial kinetic energy of $500 \mathrm{keV}$, respectively. The dependence on the initial kinetic 

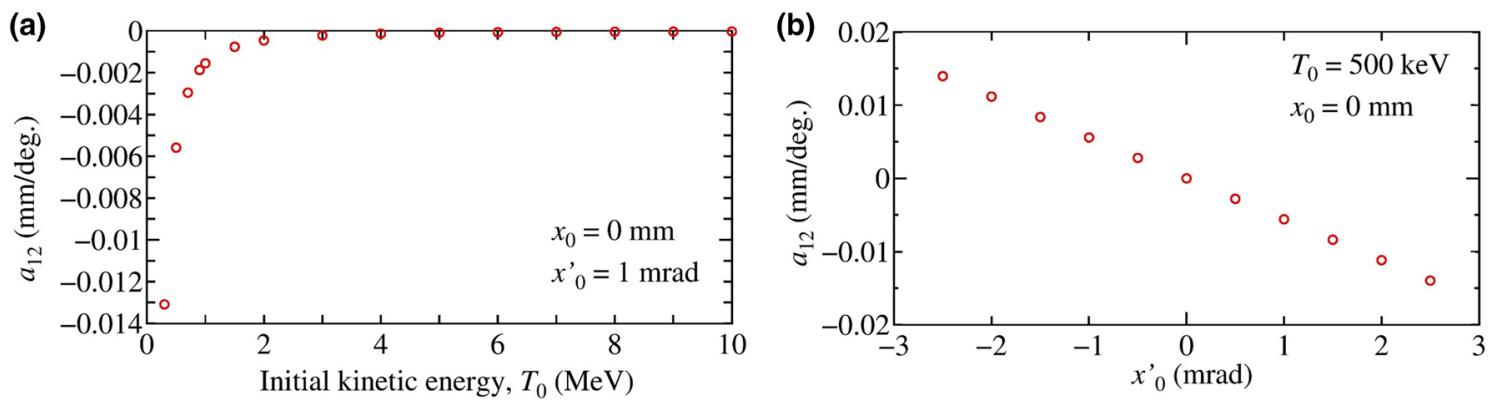

Figure 4. Deflection coefficient $a_{12}$ as functions of (a) the initial kinetic energy $T_{0}$ with the initial condition of $x_{0}=0 \mathrm{~mm}$ and $x_{0}^{\prime}=1 \mathrm{mrad}$, and (b) the initial angle $x_{0}^{\prime}$ with an initial kinetic energy of $500 \mathrm{keV}$ and an initial offset of $x_{0}=0 \mathrm{~mm}$.

energy shown in Fig. 4 indicates that the deflection effect associated with the initial angle is effective for $T_{0}<2$ $\mathrm{MeV}$, similar to the effect associated with the initial position, $x_{0}$. Then, we can define $a_{12}\left(x_{0}^{\prime}\right)=d_{12} x_{0}^{\prime}$, where $d_{12}$ is a coefficient associated with the initial angle. Thus, the deflection effect associated with the initial angle becomes:

$$
\Delta x_{d}(\Delta \phi)=d_{12} x_{0}^{\prime} \Delta \phi
$$

Deflection effect for low energy electron. Based on the results of the analysis of the deflection effect as functions of the initial position and initial angle presented in the previous section, we can summarize the deflection effect for low energy beams with small phase deviations as

$$
x_{1}=M x_{0}+\Delta \phi D x_{0},
$$

Here, $M$ is a linear transfer matrix for the on-crest phase whose elements are $m_{11}, m_{12}, m_{21}$ and $m_{22}$, and $D$ is a $2 \times 2$ matrix with the elements $d_{11}, d_{12}, d_{21}$ and $d_{22}$. $D$ indicates the strength of the deflection effect which can be determined by numerical simulations or experimental measurements. The beam position at the exit of the beamline is described by

$$
x_{1}(\Delta \phi)=x_{c}+a_{1} \Delta \phi=x_{c}+\left(d_{11} x_{0}+d_{12} x_{0}^{\prime}\right) \omega \Delta t .
$$

where $x_{c}=m_{11} x_{0}+m_{12} x_{0}^{\prime}$. The strength of the deflection effect can be controlled by $x_{0}$ and $x_{0}^{\prime}$. For bunch length measurements, the coefficient $a_{1}$ can be measured by scanning the phase offset, $\Delta \phi$. The temporal distribution of the electron bunch with respect to $\Delta t$ is projected on the transverse distribution. This only considered the deflection effect for a single electron with the time difference, $\Delta t$. Here, we consider an electron bunch with a temporal distribution $f(\tau)$ at the entrance of the beamline, and a horizontal distribution $g(x)$ at its exit. The horizontal distribution can be measured by a profile monitor located at the exit of the beamline. The parameter $\tau$, which is the time difference from the center of the electron bunch is adjusted to the maximum acceleration condition. The horizontal distribution, which can be measured using the profile monitor installed at the exit of the beam line, convoluted by the initial temporal distribution, is given by

$$
G(x)=\int f(\tau) g\left(x-\left(d_{11} x_{0}+d_{12} x_{0}^{\prime}\right) \omega \tau\right) d \tau .
$$

In order to calculate the projection of the bunch length to the transverse beam size, we assume that the electron beam in the longitudinal phase space follows a Gaussian distribution, and is transversally small compared to the transverse radius of the RF cavity. We describe the transverse beam size at the profile monitor as functions of the bunch length and accelerating RF cavity parameters as

$$
\sigma_{x}=\sqrt{\sigma_{x 0}^{2}+\left(\left(d_{11} x_{0}+d_{12} x_{0}^{\prime}\right) \omega \sigma_{t}\right)^{2}},
$$

where $\sigma_{t}$ is the rms bunch length, and $\sigma_{x 0}$ is the rms beam size when the electron bunch passes the center of the accelerating RF cavity. As a consequence of Eq. (9), the transverse beam size at the profile monitor is proportional to the square root of the bunch length. In addition, Eq. (9) represents the essence of the measurement of the bunch length using the accelerating RF cavity. The deflection coefficient $a_{1}$ is a crucial parameter because it affects the correlation between the longitudinal and transverse directions. The parameters $x_{0}$ and $x_{0}^{\prime}$ are also important for controlling the strength of the deflection effect since $a_{1}$ is a linear function of both parameters. In our method, the deflection coefficient is determined experimentally. The initial beam offset, $x_{0}$, and the angle, $x_{0}^{\prime}$, are controlled in accordance with the resolution required for the bunch length.

Resolution of bunch length measurement. The estimation of the temporal resolution is important ${ }^{54}$ for validating the proposed method. The temporal resolution, $R_{t}$, can be defined as the bunch length that yields on the profile monitor, a transverse beam size equal to the transverse resolution of the monitor itself. In this case, 


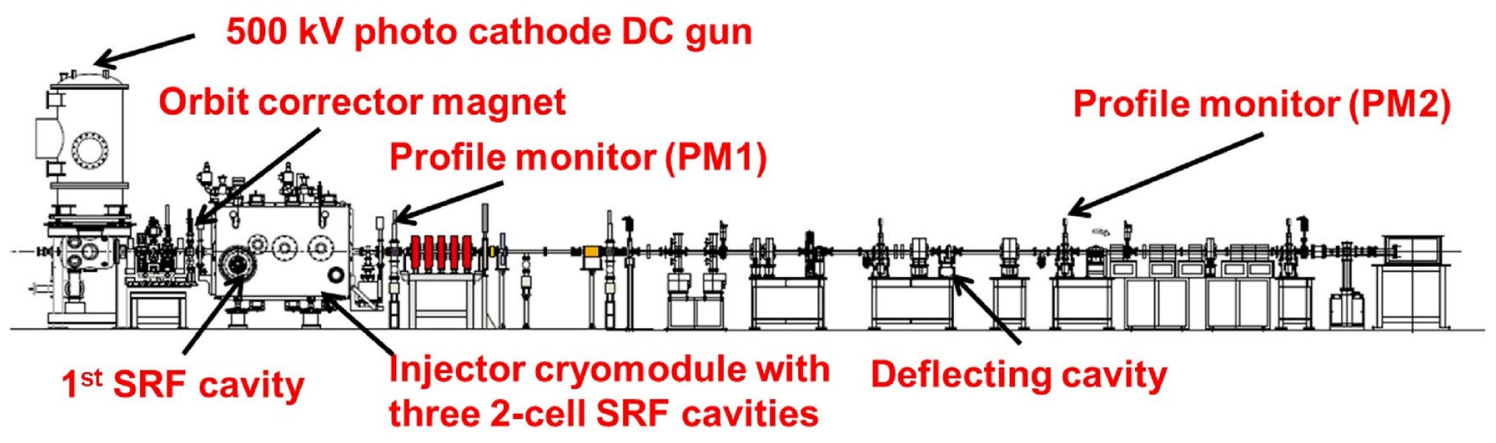

Figure 5. Layout of an injector beam line for the cERL.

the transverse resolution for the bunch length measurement is $\sigma_{x 0}+\sigma_{\mathcal{c}}$, where $\sigma_{c}$ is the transverse resolution of the beam size measured by the profile monitor. This limits the resolution of the bunch length. According to Eq. (9) the temporal resolution of the proposed method is given by

$$
R_{t}=\frac{1}{\left|d_{11} x_{0}+d_{12} x_{0}^{\prime}\right| \omega} \sqrt{\sigma_{c}^{2}+2 \sigma_{x 0} \sigma_{c}} .
$$

The temporal resolution of the proposed method depends on the deflection coefficient which, in turn, depends on the initial beam energy, amplitude of the electric field, and initial beam size, $R_{12}$, which is dominated by the distance and devices between the accelerating RF cavity and the profile monitor, and the beam offset inside the accelerating RF cavity. Among these parameters, the deflection coefficient and beam offset are important, because they can be controlled independently of the conditions of the beam transportation line. Since the deflection coefficient is determined by the design of the RF cavity, the beam offset inside it is an important parameter for controlling the temporal resolution of our method.

Since the method requires the beam offset inside the RF cavity, the kick effect owing to the transverse wakefield should be considered. The kick angle resulting from the transverse wakefield is obtained using ${ }^{55-57}$

$$
\Delta r^{\prime}(s)=\frac{e I_{0} L r}{c E_{k}} \int_{0}^{s} d s^{\prime} W_{t r}\left(s^{\prime}\right),
$$

where $L$ is the length of the beam pipe, $r$ is the beam offset from the center of the cavity, $E_{k}$ is the beam energy, $I_{0}$ is the beam current, and $W_{t r}=4 Z_{0} c s_{0} \phi(s) / \pi a^{4}\left[1-\left(1+\sqrt{s / s_{0}}\right) \exp \left(-\sqrt{s / s_{0}}\right)\right]$. In addition, $Z_{0}=120$ $\pi \Omega, a$ is the radius of the iris, $\phi(s)$ is the step function, $s_{0}=g / 8(a /(\alpha(g / L) L))^{2}$, where $g$ is the gap, and $\alpha(\gamma) \sim 1-\alpha_{1} \sqrt{\gamma}-\left(1-2 \alpha_{1}\right) \gamma$ with $\alpha_{1}=0.4648$. Although the wakefield model is valid for ultra-relativistic beams $(\beta \sim 1)$ with an elongated structure longer than the catch-up distance, it probably gives the most accurate estimation of the order of magnitude of the kick angle arising from the wakefield. In our case, the kick angle corresponding to a beam offset of $10 \mathrm{~mm}$, bunch charge of $10 \mathrm{fC}$, iris radius of $3.37 \mathrm{~cm}$, bunch length of $3 \mathrm{ps,}$ and energy of $0.39 \mathrm{MeV}$ is lower than $0.04 \mu \mathrm{rad}$, i.e., negligible. It was verified experimentally that the emittance growth owing to the radial electric-field is significantly greater than the contribution by long-range and short-range wakefield effects ${ }^{58}$.

Experimental setup at cERL. This technique is demonstrated in a high-performance cERL injector ${ }^{59}$, which consists of a $500 \mathrm{kV}$ photo cathode DC gun ${ }^{60-62}$ and three 2-cell SRF cavities ${ }^{63,64}$. The cERL injector can produce electron bunches with a repetition rate of $1.3 \mathrm{GHz}$ and bunch charges from a few fC to pC. Macro-pulses have burst lengths of 0.1-1.2 $\mu$ s and repetition rate of $5 \mathrm{~Hz}^{10}$. The layout of the cERL injector is shown in Fig. 5. The deflection coefficient $a_{1}$ of the cavity can be determined experimentally by measuring the relation between the RF phase of the cavity and the displacement of the central position at a profile monitor $X_{c}$ installed downstream in the cavity. Using Eq. (4), the coefficient can be represented as $a_{1}=\Delta x_{d}(\Delta \phi) / \Delta \phi=\left(d_{11} x_{0}+d_{12} x_{0}^{\prime}\right)$. Since we introduce the initial beam angle $x_{0}^{\prime}$ by adjusting the strength of the corrector magnet installed upstream in the cavity during the experiment, the coefficient can be approximated to $a_{1}=\left(d_{12} x_{0}^{\prime}\right)$. The schematic layout for measuring the deflection coefficient is depicted in Fig. 6.

Since the deflection coefficient is linearly proportional to the initial beam angle $x_{0}^{\prime}$, offset $x_{0}$, and effective accelerating field of the cavity, the beam and machine parameters are evaluated carefully. During calibration, the gun voltage was set to $390 \mathrm{kV}$ and provide a $3.3 \mathrm{ps}$ (rms) laser pulse on the GaAs cathode to produce a $10 \mathrm{fC}$ charged electron bunch ${ }^{65}$. After tuning the gun component of the injector, the corrector magnet used to control the beam offset inside the RF cavity was calibrated using the profile monitor (PM1) installed in the downstream of the cavity as shown in Fig. 5. Next, the strength of the corrector magnet was set to the beam passing the accelerating RF cavity with a reasonable beam offset, $7.85 \mathrm{~mm}^{58}$, and the beam position was measured at the profile monitor while the phase of the accelerating RF cavity was changed.

Figure 7 shows the result of the measurement of the displacement as a function of the RF phase. These measurements were used to calibrate the coefficient $d_{12}=a_{1} / x_{0}^{\prime}$, yielding a value of $-0.0324 \pm 0.00026 \mathrm{~mm} / \mathrm{deg} /$ mrad. Under this condition, the beam is accelerated from $390 \mathrm{keV}$ to $1.86 \mathrm{MeV}$. 


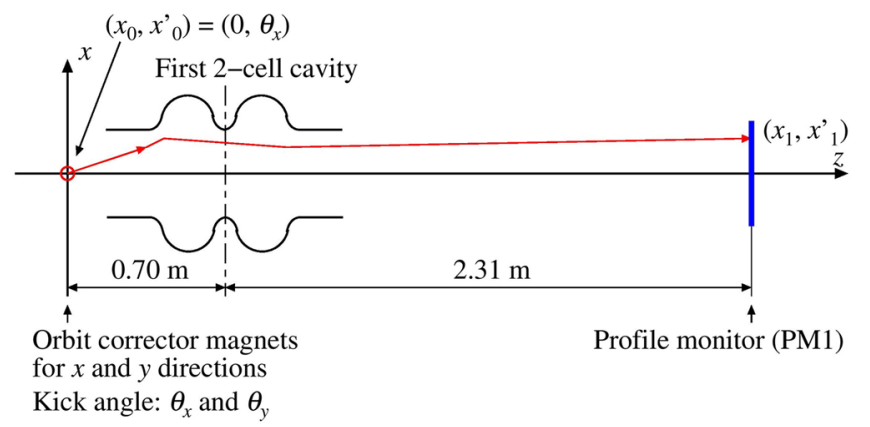

Figure 6. Schematic view of the bunch length measurement in the cERL injector. Corrector magnets for the horizontal and vertical directions, located at the entrance, control the kick angles $\theta_{x}$ and $\theta_{y}$. The transverse profile is measured by a profile monitor (PM1).

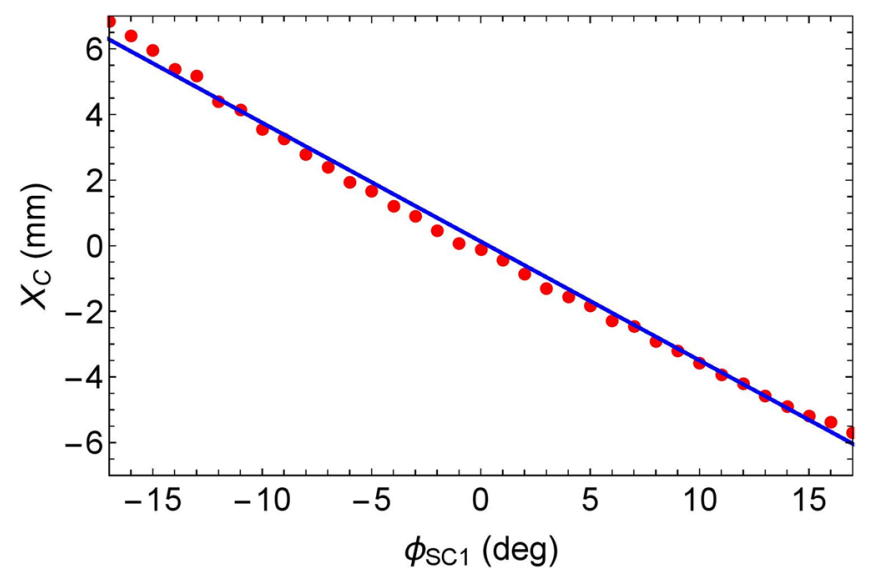

Figure 7. Result of the measurement of the displacement of the beam position at the profile monitor as a function of the RF phase.

A reasonable beam offset that matces the resolution required for the bunch length measurement by our system can be estimated based on the calibrated deflection coefficient. In our system, the resolution of the transverse beam size measurement using the profile monitor $\sigma_{c}$ is smaller than $0.10 \mathrm{~mm}$, and the initial beam size when the electron beam passes the center of the accelerating RF cavity, $\sigma_{x 0}$, is $0.469 \mathrm{~mm}$. These these parameters were used to calculate the resolution as a function of the beam offset based on Eq. (10). In order to measure the bunch length of $3.3 \mathrm{ps}(\mathrm{rms})$, the beam offset inside the accelerating RF cavity should be larger than $4.80 \mathrm{~mm}$. The energy variation by the phase shift due to the beam offset is negligible. We note that the beam offset should be kept as small as possible during the experiment to avoid the effects of the transverse wakefield and non-linearity for a large offset even though it controls the resolution significantly. In our case, the maximum beam offset is limited by the inner radius of the RF cavity, which is about $35 \mathrm{~mm}$. Assuming that the initial beam angle is $16 \mathrm{mrad}$, corresponding to a beam offset equivalent to $1 / 3$ of the cavity radius, the resolution of the proposed method is estimated to be about 0.98 ps using Eq. (10). The resolution can be improved by increasing the value of $d_{12}$ coefficient which can be achieved by increasing the field gradient of the cavity or lowering the initial beam energy.

\section{Results}

We demonstrated the projection of the longitudinal profile to the transverse direction by an accelerating RF cavity with a beam offset inside it by measuring of the temporal profile with a long bunch, in which eight Gaussian pulses with a bunch length of $3.3 \mathrm{ps}$ (rms) were stacked. In the experiment, the first cavity was turned on and the peak on-axis accelerating field of the accelerating RF cavity, $E_{0}$, was set to $7.21 \mathrm{MV} / \mathrm{m}$. The second and third cavities were turned off, and the beam corrector magnet, installed upstream in the cavity, was adjusted to produce horizontal or vertical beam offsets of $16 \mathrm{~mm}$ from the electromagnetic center of the cavity. The beamline is a non-dispersive section. Since the electron beam produced by the DC gun has a energy spread of lower than $0.01 \%$, the dispersion caused by the corrector magnet in the beamline, which leads to variations in the beam size, can also be ignored. The electromagnetic center of the cavity was estimated by measuring the $d X_{C} / d \phi$ and $d Y_{C} / d \phi$ values that were derived in Ref. ${ }^{58}$.

The top graph of Fig. 8 shows the transverse profile measured by the profile monitor (PM1) for zero offset inside the cavity. In this case, the beam passed through the electromagnetic center of the cavity, and the measured 

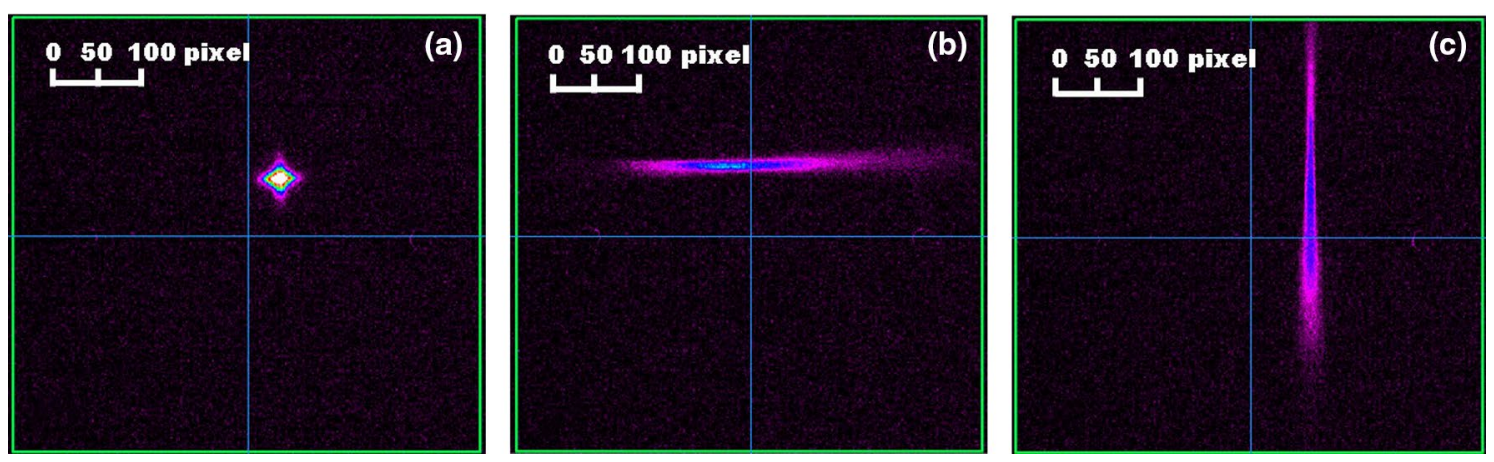

Figure 8. Measured transverse beam profiles with a bunch length of $13.51 \mathrm{ps}$ (rms) for (a) zero offset, (b) nonzero horizontal beam offset, $\Delta x=16.1 \mathrm{~mm}$, and (c) non-zero vertical beam offset, $\Delta y=16.0 \mathrm{~mm}$ in the cavity. Each pixel on the profile monitor corresponds to $54.9 \mu \mathrm{m}$.

(a)

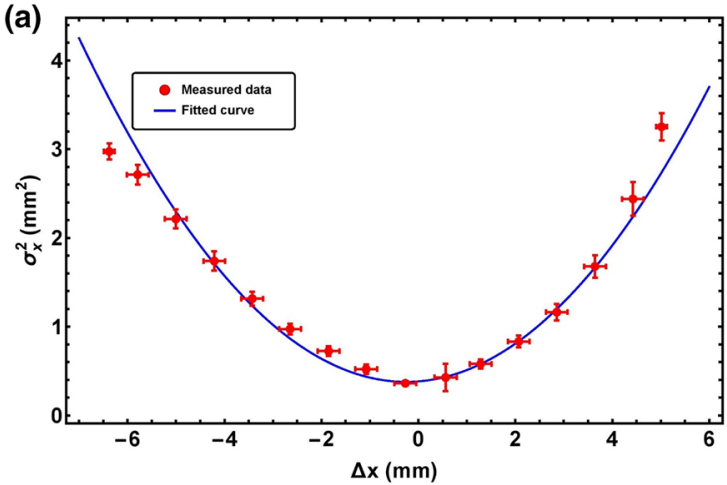

(b)

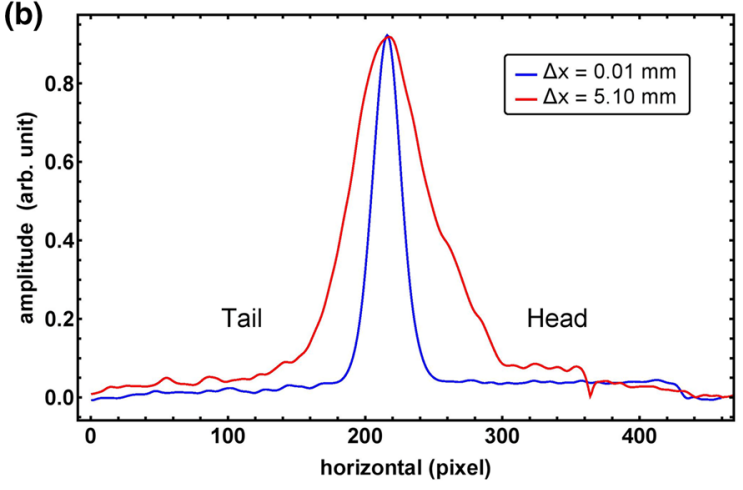

Figure 9. Result of the measurement of (a) the relation between the horizontal beam offset and the beam size at the profile monitor for a long bunch and (b) temporal profile with beam offsets of $0.01 \mathrm{~mm}$ and $5.1 \mathrm{~mm}$.

profile was almost same as the original transverse beam size. This shows a lack of correlation between the longitudinal and transverse directions owing to the deflecting force. This also indicates that the electromagnetic center of the cavity was accurately estimated by measuring $d X_{C} / d \phi$ and $d Y_{C} / d \phi^{58}$. The lower graphs of Fig. 8 show the transverse profiles with horizontal or vertical offsets of $16 \mathrm{~mm}$ from the center. In these cases, the longitudinal distribution was projected to the horizontal or vertical direction. These results show that the non-zero beam offset inside the cavity causes the correlation between the longitudinal and transverse directions, thereby validating the principle of our method. Moreover, the variation of the beam size variation as a function of the beam offset inside the cavity was measured to estimate the length of the temporal profile. The result is shown in Fig. 9. The minimum beam size corresponds to the zero offset case.

In order to analyze the measured data, Eq. (9) can be expressed as

$$
\sigma_{x}^{2}=\sigma_{x 0}^{2}+\xi(\Delta x+\chi)^{2}
$$

where $L_{0}$ is the distance between the corrector magnet and the center of the cavity, $\Delta x$ is the beam offset at the center of the cavity, and $\chi$ is a constant that represents the minimum beam size position. Since this method measures a relative beam size change respect to $\sigma_{0}$ in Eq. (12) which is determined by the integration of complex physics processes including optics, energy variation as well as low-energy beam dynamics, it is no longer necessary to deconvolute the emittance dilution by space charge effects. The $\xi$ can be represented as $\xi=\left(d_{12} \omega \sigma_{z} / L_{0}\right)^{2}$ because the beam is aligned to the center of cavity $x_{0}=0$. Using the fitted curve plotted in Fig. 9 , which is derived from Eq. (12), the $\xi$ value was calculated to be $0.0850 \pm 0.000348$. The beamline parameters involves the conversion of the variation of the beam size as a function of the beam offset inside the cavity to the rms bunch length. In this experiment, the $E_{p}, E_{c}, R_{12}$, and $\Delta \phi$ were $1.86 \mathrm{MeV}, 0.39 \mathrm{MeV}, 2.308 \mathrm{~m}$, and $0^{\circ}$, respectively. The parameters $E_{c}$ and $E_{p}$ are the beam energies at the entrance of the RF cavity and profile monitor, respectively. Using these parameters, our method yielded a bunch length of $13.51 \pm 0.03 \mathrm{ps}(\mathrm{rms})$.

Measurement for a few-ps bunch. In the previous section, the principle of our method was confirmed by measuring the temporal profile for a the long bunch length. It is necessary to verify the accuracy of our method for few-ps bunch lengths. We then measured the bunch length for a single Gaussian pulse whose bunch length is $3.3 \mathrm{ps}(\mathrm{rms})$. Measurements have been performed at the peak on-axis accelerating field $E_{\text {acc }}$ of 7.95 $\mathrm{MV} / \mathrm{m}(\Delta E=1.62 \mathrm{MeV})$ and $7.11 \mathrm{MV} / \mathrm{m}(\Delta E=1.45 \mathrm{MeV})$ since the peak on-axis accelerating field strength 

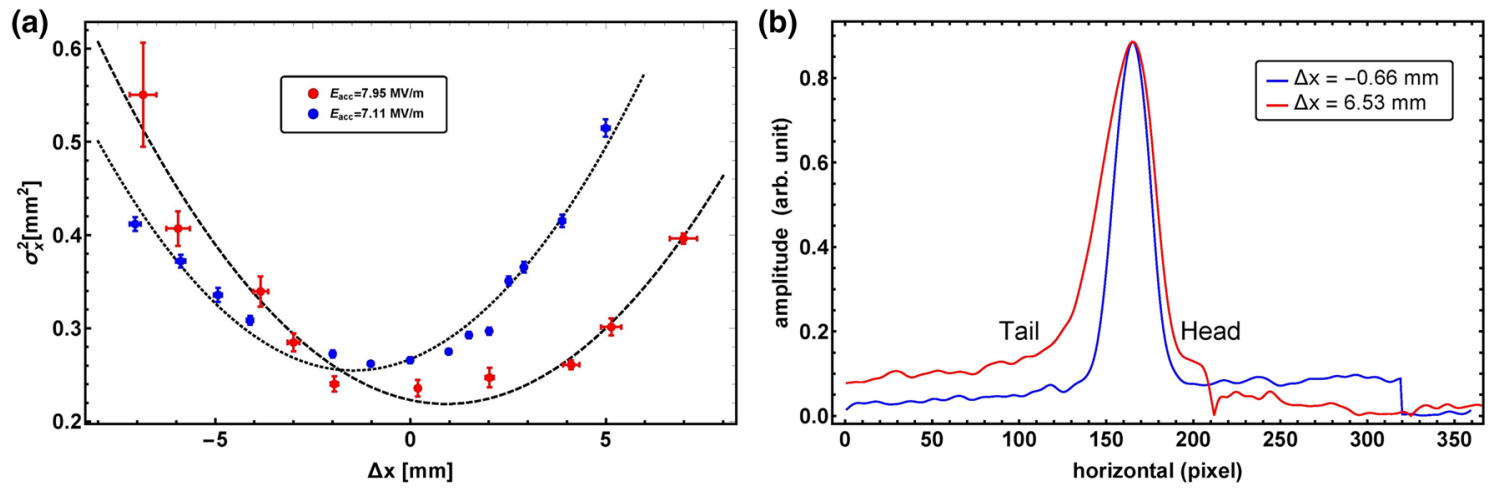

Figure 10. Result of the measurements of (a) the relation between the horizontal beam offset and beam size at the profile monitor with peak on-axis accelerating fields of 7.11 and $7.95 \mathrm{MV} / \mathrm{m}$, and (b) temporal profile with beam offsets of $-0.66 \mathrm{~mm}$ and $6.53 \mathrm{~mm}$. This shows a long tail produced by the GaAs cathode.

influences on the accuracy of the bunch length measurement. The beam offset inside the accelerating RF cavity should exceed $4.80 \mathrm{~mm}$ to measure a bunch length of $3.3 \mathrm{ps}(\mathrm{rms})$. The results are presented in Fig. 10.

Using the measured result, the value of $\xi$ was estimated as $0.00488 \pm 0.000471$ for $E_{a c c}=7.95 \mathrm{MV} / \mathrm{m}$ and $3.39 \pm 0.12 \mathrm{ps}(\mathrm{rms})$ for $E_{a c c}=7.11 \mathrm{MV} / \mathrm{m}$. The bunch length was calculated to be $2.94 \pm 0.15 \mathrm{ps}$ ( $\mathrm{rms}$ ) for $E_{a c c}$ $=7.95 \mathrm{MV} / \mathrm{m}$ and $0.00558 \pm 0.000389$ for $E_{a c c}=7.11 \mathrm{MV} / \mathrm{m}$. This result is consistent with expectations because the duration of the laser pulse on the photo cathode is $3.3 \mathrm{ps}(\mathrm{rms})^{65}$. The result of the bunch length measurement with a lower accelerating gradient also agrees with the duration of the laser pulse on the photo cathode. Thus, the results demonstrate that the proposed method is suitable for few-ps bunch lengths. Since the beam angle is varied up to $8.5 \mathrm{mrad}$ using the corrector magnet to achieve a beam offset of $6 \mathrm{~mm}$ at the center of the cavity, it causes variations of the beam trajectory at the entrance and exit of the cavity. This effect leads to a shift in the minimum beam size position and an increase in the negative horizontal deviation of the measured points from the fitted curve for large $\sigma_{x}^{2}$.

In conclusion, we have proposed and demonstrated an experimental method for measuring few-ps level bunch lengths using the deflecting force of an accelerating RF cavity, whose main function is to increase the beam energy. The ability to perform few-ps level bunch length measurements was validated experimentally using a 2-cell $1.3 \mathrm{GHz}$ SRF cavity in the cERL injector at KEK. Bunch lengths of $3.3 \mathrm{ps}$ (rms) with a bunch charge of $10 \mathrm{fC}$ were measured accurately using the cavity, under the influence of an acceleration field of $7.21 \mathrm{MV} / \mathrm{m}$. The measured values agree strongly with the rms duration of the laser pulse on the photo cathode. The proposed method can be applied to the normal-conducting injectors of the many accelerators worldwide which have an S-band cavity with low beam energy because the S-band cavities yield a higher gradient. The most critical aspect towards a sub-ps resolution at high bunch charge involves the nonlinear effects of wakefield and space-charge. It is necessary to estimate carefully the contribution of these effects on the transverse beam profile ${ }^{66}$.

Received: 4 August 2020; Accepted: 20 October 2020

Published online: 03 November 2020

\section{References}

1. Rossbach, J. et al. A VUV free electron laser at the TESLA test facility at DESY. Nucl. Instrum. Methods Phys. Res. Sect. A 375, 269 (1996).

2. Emma, P. et al. First lasing and operation of an ångstrom-wavelength free-electron laser. Nat. Photon. 4, 641 (2010).

3. Kim, E.-S., Hwang, J.-G. \& Yoon, M. Parameter optimizations and performances for the low-charge beams in PAL free-electron laser. IEEE Trans. Nucl. Sci. 58, 2000 (2011).

4. Ishikawa, T. et al. A compact X-ray free-electron laser emitting in the sub-ångström region. Nat. Photon. 6, 540 (2012).

5. Kang, H.-S. et al. Hard X-ray free-electron laser with femtosecond-scale timing jitter. Nat. Photon. 11, 708 (2017).

6. Halavanau, A., Decker, F.-J., Emma, C., Sheppard, J. \& Pellegrini, C. Very high brightness and power LCLS-II hard X-ray pulses. J. Synchrotron Radiat. 26, 635 (2019).

7. Decking, W. et al. A MHz-repetition-rate hard X-ray free-electron laser driven by a superconducting linear accelerator. Nat. Photon. 14, 391 (2020).

8. Neil, G. R. et al. Sustained kilowatt lasing in a free-electron laser with same cell energy recovery. Phys. Rev. Lett. 84, 662 (2000).

9. Bilderback, D. H. Energy recovery linac (ERL) coherent hard x-ray sources. New J. Phys. 12, 035011 (2010).

10. Akemoto, M. et al. Construction and commissioning of the compact energy-recovery linac at KEK. Nucl. Instrum. Methods Phys. Res. Sect. A 877, 197 (2018).

11. Bartnik, A. et al. CBETA: First multipass superconducting linear accelerator with energy recovery. Phys. Rev. Lett. 125, 044803 (2020).

12. Behnke, T., Brau, J. E., Foster, B., Fuster, J., Harrison, M., et al. The International Linear Collider Technical Design Report-Volume 1: Executive Summary. arXiv:1306.6327 [physics.acc-ph] (2013).

13. Balazs, C., Charles, T. K. \& CLIC and CLICdp collaborations. Updated baseline for a staged compact linear collider. CERN Yellow Report; Vol. CERN-2016-004. (CERN, 2016). https://doi.org/10.5170/CERN-2016-004.

14. Michizono, S. The International Linear Collider. Nat. Rev. Phys. 1, 244. https://doi.org/10.1038/s42254-019-0044-4 (2019).

15. Sicking, E. \& Ström, R. From precision physics to the energy frontier with the Compact Linear Collider. Nat. Phys. 16, 386 (2020). 
16. Dowell, D. H. et al. First operation of a photocathode radio frequency gun injector at high duty factor. Appl. Phys. Lett. 63, 2035 (1993).

17. Janssen, D. et al. First operation of a superconducting RF gun. Nucl. Instrum. Methods Phys. Res. Sect. A 507, 314 (2003).

18. Arnold, A. et al. Development of a superconducting radio frequency photoelectron injector. Nucl. Instrum. Methods Phys. Res. Sect. A 577, 440 (2007)

19. Arnold, A. \& Teichert, J. Overview on superconducting photoinjectors. Phys. Rev. ST Accel. Beams 14, 024801 (2011).

20. Gulliford, C. et al. Demonstration of low emittance in the Cornell energy recovery linac injector prototype. Phys. Rev. ST Accel. Beams 16, 073401 (2013).

21. Teichert, J. et al. Free-electron laser operation with a superconducting radio-frequency photoinjector at ELBE. Nucl. Instrum. Methods Phys. Res. Sect. A 743, 114 (2014).

22. Pierini, P. et al. Fabrication and vertical test experience of the European X-ray Free Electron Laser $3.9 \mathrm{GHz}$ superconducting cavities. Phys. Rev. ST Accel. Beams 20, 042006 (2017).

23. Kim, S.-Y. et al. Commissioning of the electron injector for the AWAKE experiment. Nucl. Instrum. Methods Phys. Res. Sect. A 953, $163194(2020)$.

24. White, W. E., Robert, A. \& Dunne, M. The linac coherent light source. J. Synchrotron Radiat. 22, 472 (2015).

25. Hartmann, N. et al. Attosecond time-energy structure of X-ray free-electron laser pulses. Nat. Photon. 12, 215 (2018).

26. Kamps, T. et al. Scientific Opportunies for bERLinPro 2020+, Report with Ideas and Conclusions from bERLinProCamp 2019. arXiv:1910.00881 [physics.acc-ph] (2019).

27. Carr, G. et al. High-power terahertz radiation from relativistic electrons. Nature 420, 153 (2002)

28. Green, B. et al. High-field high-repetition-rate sources for the coherent THz control of matter. Sci. Rep. 6, 22256 (2016).

29. Lumpkin, A. H., Yang, B. X. \& Chae, Y. C. Observations of bunch-lengthening effects in the APS 7-GeV storage ring. Nucl. Instrum. Methods Phys. Res. Sect. A 393, 50 (1997).

30. Schiwietz, G., Hwang, J.-G., Koopmans, M., Ries, M. \& Schälicke, A. Development of the electron-beam diagnostics for the future BESSY-VSR storage ring. J. Phys.: Conf. Ser. 1067, 072005 (2018).

31. Schiwietz, G., Hwang, J.-G., Koopmans M. \& Ries, M. Overview of bunch-resolved diagnostics for the future BESSY VSR electronstorage ring. In Paper presented a the 8th International Beam Instrumentation Conference. Proceedings of IBIC 2019, MOCO04, Malmö, Sweden (2019).

32. Akre, R. et al. Bunch length measurements using a transverse RF deflecting structure in the SLAC linac. Paper presented at the $8^{\text {th }}$ European Particle Accelerator Conference. Proceedings of EPAC 2002, 1882, Paris, France (2002).

33. Floettmann, K. \& Paramonov, V. Beam dynamics in transverse deflecting rf structures. Phys. Rev. ST Accel. Beams 17, 024001 (2014).

34. Maxson, J. et al. Direct measurement of sub-10 fs relativistic electron beams with ultralow emittance. Phys. Rev. Lett. 118, 154802 (2017).

35. Paramonov, V., Danielyan, V., Floettmann, K., Simonyan, A. \& Tsakanov, V. ,. Design, construction and tuning of an RF deflecting cavity for the REGAE facility. J. Phys.: Conf. Ser. 1238, 012069 (2019).

36. Marx, D. et al. Simulation studies for characterizing ultrashort bunches using novel polarizable X-band transverse deflection structures. Sci. Rep. 9, 19912 (2019).

37. Wilke, I. et al. Single-shot electron-beam bunch length measurements. Phys. Rev. Lett. 88, 124801 (2002).

38. Berden, G. et al. Electro-optic technique with improved time resolution for real-time, nondestructive, single-shot measurements of femtosecond electron bunch profiles. Phys. Rev. Lett. 93, 114802 (2004).

39. Zhao, L. et al. Terahertz streaking of few-femtosecond relativistic electron beams. Phys. Rev. X 8, 021061 (2018).

40. Li, R. K. et al. Terahertz-based subfemtosecond metrology of relativistic electron beams. Phys. Rev. Accel. Beams 22, 012803 (2019).

41. Wang, D. X., Krafft, G. A. \& Sinclair, C. K. Measurement of femtosecond electron bunches using a rf zero-phasing method. Phys. Rev. E 57, 2283 (1998).

42. Kung, P., Lihn, H. C., Wiedemann, H. \& Bocek, D. Generation and measurement of 50-fs (rms) electron pulses. Phys. Rev. Lett. 73, 967 (1994).

43. Murokh, A. et al. Bunch length measurement of picosecond electron beams from a photoinjector using coherent transition radiation. Nucl. Instrum. Methods Phys. Res. Sect. A 410, 452 (1998).

44. Leissner, B. et al. Bunch Length Measurements using a Martin Puplett Interferometer at the TESLA Test Facility Linac. Paper presented at the 18th Particle Accelerator Conference. Proceedings of PAC 1999, 2172, New York, USA (1999).

45. Korbly, S. E., Kesar, A. S., Temkin, R. J. \& Brownell, J. H. Measurement of subpicosecond bunch lengths using coherent SmithPurcell radiation. Phys. Rev. ST Accel. Beams 9, 022802 (2006).

46. Muller, A. -S. et al. Characterizing THz Coherent Synchrotron Radiation at the ANKA Storage Ring. Paper presented at the 14th European Particle Accelerator Conference. Proceedings of EPAC 2008, WEPC047, Genoa, Italy (2008).

47. Casalbuoni, S., Schmidt, B., Schmuser, P., Arsov, V. \& Wesch, S. Ultrabroadband terahertz source and beamline based on coherent transition radiation. Phys. Rev. ST Accel. Beams 12, 030705 (2009).

48. Veronese, M., Appio, R., Craievich, P. \& Penco, G. Observation of gigawatt-class THz pulses from a compact laser-driven particle accelerator. Phys. Rev. Lett. 110, 074802 (2013).

49. Rosenzweig, J. \& Serafini, L. Transverse particle motion in radio-frequency linear accelerators. Phys. Rev. E 49, 1599 (1994).

50. Energy Recovery Linac Conceptual Design Report. KEK Report 2012-4, KEK, October, 2013. http://ccdb5fs.kek.jp/ tiff/2012/1224/1224004.pdf.

51. Kim, K. J. RF and space-charge effects in laser-driven RF electron Guns. Nucl. Instrum. Methods Phys. Res. Sect. A 275, 201 (1989).

52. Pulsar Physics, General Particle Tracer. http://www.pulsar.nl/gpt.

53. Halbach, K. \& Holsinger, R. F. Superfish-a computer program for evaluation of RF cavities with cylindrical symmetry. Part. Accel. 7, $213(1976)$.

54. Alesini, D. et al. RF deflector design and measurements for the longitudinal and transverse phase space characterization at SPARC. Nucl. Instrum. Methods Phys. Res. Sect. A 568, 488 (2006).

55. Bane, K.L.F., Mosnier, A., Novokhatski, A., Yokoya, K. Calculations of the short-range longitudinal wakefields in the NLC linac. Paper presented at the 1998 International Computational Accelerator Physics Conference. Proceedings of. ICAP, CTH12 1998 (Monterey, CA, USA, 1998).

56. Bane, K.L.F. Short Range Wakefields in Accelerating Structures for the NLC. SLAC-PUB-9663 (2003).

57. Karlberg, O. et al. Short range wakefields in MAX IV and FERMI Linac. Paper presented at the $3^{\text {rd }}$ International Particle Accelerator Conference. Proceedings of IPAC. WEPPR060 2012 (New Orleans, Louisiana, USA, 2012).

58. Hwang, J.-G. et al. Analysis of effects of transverse electric field in an injector cavity of compact-ERL at KEK. Nucl. Instrum. Methods Phys. Res. Sect. A 753, 97 (2014).

59. Miyajima, T. et al., Beam Dynamics Simulation for the Compact ERL Injector. Paper presented at the 23rd Particle Accelerator Conference. Proceedings of PAC 2009, MO6RFP067, Vancouver, Canada (2009).

60. Nishimori, N. et al. Development of a 500-kV Photocathode DC Gun for ERLS. J. Phys.: Conf. Ser. 298, 012005 (2011).

61. Nishimori, N. et al. Generation of a 500-keV electron beam from a high voltage photoemission gun. Appl. Phys. Lett. 102, 234103 (2013). 
62. Yamamoto, M. \& Nishimori, N. High voltage threshold for stable operation in a dc electron gun. Appl. Phys. Lett. 109, 014103 (2016).

63. Watanabe K. et al., SC cavity system for ERL injector at KEK. Paper presented at the 14th International Conference on RF Superconductivity. Proceedings of SRF2009, TUPPO056, Berlin, Germany (2009).

64. Watanabe, K., Noguchi, S., Kako, E., Umemori, K. \& Shishido, T. Development of the superconducting rf 2-cell cavity for cERL injector at KEK. Nucl. Instrum. Methods Phys. Res. Sect. A 714, 67 (2013).

65. Sakanaka, S. et al. Construction and Commissioning of Compact-ERL Injector at KEK. Paper presented at the 53rd ICFA Advanced Beam Dynamics Workshop on Energy Recovery Linacs. Proceedings of ERL-2013 Workshop, WG102, Novosibirsk, Russia (2013).

66. Hwang, J.-G., Kim, E.-S. \& Miyajima, T. Effects of space charge in a compact superconducting energy recovery linac with a low energy. Nucl. Instrum. Methods Phys. Res. Sect. A 684, 18 (2012).

\title{
Acknowledgements
}

The authors wish to thank their collaborators at cERL, Takashi Obina, Ryota Takai, Eiji Kako, Shinichiro Michizono, Takako Miura, Qiu Feng, Kentaro Harada, Miho Shimada, Tatsuya Kume, Shinya Nagahashi, Norio Nakamura, Shogo Sakanaka, Masahiro Yamamoto and Takashi Uchiyama at the High Energy Accelerator Research Organization (KEK), and Ryoichi Hajima, Ryoji Nagai and Nobuyuki Nishimori at the Japan Atomic Energy Agency (JAEA), for their efforts in constructing and performing the machine commissioning. In addition, this work was supported by the German Bundesministerium für Bildung und Forschung, Land Berlin and grants of Helmholtz Association and the National Research Foundation of Korea (NRF) funded by the Ministry of Education, Science and Technology (MEST) (Center for Korean J-Parc Users, Grant No. NRF-2013K1A3A7A06056592).

\section{Author contributions}

J.-G.H. and T.M. conceived the proposed scheme and performed the numerical simulations. J.-G.H., T.M., and Y.H. performed beam experiments at compact ERL. J.-G.H., T.M., and E.-S.K. analysed the results of numerical simulation and beam experiments. J.-G.H., T.M., and E.-S.K. wrote the main manuscript text. J.-G.H., and T.M. prepared figures. All authors reviewed the manuscript.

\section{Competing interests}

The authors declare no competing interests.

\section{Additional information}

Supplementary information is available for this paper at https://doi.org/10.1038/s41598-020-76054-w.

Correspondence and requests for materials should be addressed to T.M. or E.-S.K.

Reprints and permissions information is available at www.nature.com/reprints.

Publisher's note Springer Nature remains neutral with regard to jurisdictional claims in published maps and institutional affiliations.

\begin{abstract}
(c) (1) Open Access This article is licensed under a Creative Commons Attribution 4.0 International cc) License, which permits use, sharing, adaptation, distribution and reproduction in any medium or format, as long as you give appropriate credit to the original author(s) and the source, provide a link to the Creative Commons licence, and indicate if changes were made. The images or other third party material in this article are included in the article's Creative Commons licence, unless indicated otherwise in a credit line to the material. If material is not included in the article's Creative Commons licence and your intended use is not permitted by statutory regulation or exceeds the permitted use, you will need to obtain permission directly from the copyright holder. To view a copy of this licence, visit http://creativecommons.org/licenses/by/4.0/.
\end{abstract}

(c) The Author(s) 2020 